\title{
Comparison of singular spectrum analysis forecasting algorithms for student's academic performance during COVID-19 outbreak
}

\author{
Muhammad Fakhrullah Mohd Fuad ${ }^{1}$, Shazlyn Milleana Shaharudin ${ }^{*}$, Shuhaida Ismail ${ }^{2}$, Nor Ain \\ Maisarah Samsudin ${ }^{1}$ and Muhammad Fareezuan Zulfikri ${ }^{1}$ \\ Universiti Pendidikan Sultan Idris, Department of Mathematics, Faculty of Science and Mathematics, Malaysia ${ }^{1}$ \\ Department of Mathematics and Statistics, Faculty of Applied Sciences and Technology, Universiti Tun Hussein \\ Onn Malaysia, Malaysia ${ }^{2}$
}

Received: 9-November-2020; Revised: 24-January-2021; Accepted: 26-January-2021

(C)2021 Muhammad Fakhrullah Mohd Fuad et al. This is an open access article distributed under the Creative Commons Attribution (CC BY) License, which permits unrestricted use, distribution, and reproduction in any medium, provided the original work is properly cited.

\begin{abstract}
Due to the spread of COVID-19 that hit Malaysia, all academic activities at educational institutions including universities had to be carried out via online learning. However, the effectiveness of online learning is remains unanswered. Besides, online learning may have a significant impact if continued in the upcoming academic sessions. Therefore, the core of this study is to predict the academic performance of undergraduate students at one of the public universities in Malaysia by using Recurrent Forecasting-Singular Spectrum Analysis (RF-SSA) and Vector Forecasting-Singular Spectrum Analysis (VF-SSA). The key concept of the predictive model is to improve the efficiency of different types of forecast model in SSA by using two parameters which are window length $(L)$ and number of leading components (r). The forecasting approaches in SSA model was based on the Grading Point Assessments (GPA) for undergraduate students from Faculty Science and Mathematics, UPSI via online classes during COVID-19 outbreak. The experiment revealed that parameter $L=11(T / 20)$ has the best prediction result for RF-SSA model with RMSE value of 0.19 as compared to VF-SSA of 0.30 . This signifies the competency of RF-SSA in predicting the students' academic performances based on GPA for the upcoming semester. Nonetheless, an RF-SSA algorithm should be developed for higher effectivity of obtaining more data sets including more respondents from various universities in Malaysia.
\end{abstract}

Keywords

Prediction, Academic performance, COVID-19, Online learning, Singular spectrum analysis, Recurrent forecasting.

\section{Introduction}

On 31 December 2019, the Wuhan Municipal Health Commission wrote in their website about the discovery of the novel corona virus which was later confirmed by the World Health Organization (WHO) that the virus originated from Republic of China. The contagious virus namely COVID-19 can cause respiratory problems to humans infected by it. Since then, almost all countries around the world have had cases of this virus. Malaysia's first case of COVID-19 was detected on January 24, 2020 [1]. Until November 9, 2020 the total number of confirmed cases are 50,030,121 that infected in 219 countries [2].

*Author for correspondence

178
As a precaution in the dangerous virus spreading control, Tan Sri Muhyiddin bin Yassin, Malaysia's prime minister has imposed the Movement Control Order (MCO) from 18th of March, 2020 until year end. This order strictly prohibits Malaysians from engaging in any religious, sports, social and cultural activities involving many people.

Due to the MCO, all educational institutions such as preschools, primary schools, secondary schools, and higher educational institutions had to be temporarily closed. On May 27, 2020, Malaysian Ministry of Higher Education (MOHE) recommended that all higher educational institutions to continue teaching and learning activities via online platforms until the end of 2020 [3]. Online learning is any form of teaching and learning delivered through digital technology such as Google Meet, MOOC, Zoom, YouTube and etc [4]. Teaching and learning 
materials presented using these platforms have visual graphics, words, animation, video or audio to ensure the students have access to knowledge.

In Malaysia, online learning is completely rare except for blended learning which is a combination of offline and online learning. However, online learning had to be carried out entirely at all universities in Malaysia and has been going on for almost a year since the MCO was enforced. With this online learning, some of the deficiencies that can be identified which includes lack of self-motivation to learn, poor time management, unfit online learning environment, family issues [5] and various technical problems. These factors are said to cause the student's academic performance to decline and thus will affect their future. However, to what extent can the validity of these factors cause the students' academic performance to drop? There are several studies related to educational studies that highlighted COVID-19. Students in the medical field such as the radiology trainee, medical students and dental students are among those who had a huge impact on their study because they need to focus more on practical-based learning more than the theory [6-11]. There are various predictive model types that use analytics models in assessing the historical data, discovering configurations, observing trends and using that information to draft $\mathrm{p}$ future forecasts trends. These models use different type of algorithms to perform its own specific functions [12]. There are several studies used Machine Learning (ML) approach to forecast and predict student's academic performance in various levels [13-15]. However, these ML models focus on predicting the students' performance rather than overseeing, finding and analysing the trend of student's performance, level of self-motivation and identifying contributing factors that affect academic performance in order to ensure the student's readiness towards online learning.

The objectives of this paper are to (i) predict the academic performance of undergraduate students at one of the public universities in Malaysia by using Recurrent Forecasting-Singular Spectrum Analysis (RF-SSA) and Vector Forecasting-Singular Spectrum Analysis (VF-SSA) (ii) present the comparison between two forecasting algorithms. The forecasting models were applied to predict student's academic performance during online learning based on Grade Point Assessments (GPA). The findings from this study are expected to help the university in improving their online academic activities as well as identifying academically-low student achievers in advance and consequently, providing them support.

\section{Literature review}

GPA is the most emphasized thing in most universities as it is an analytical measure that shows the academic performance of students regardless of the students' mastery in their studies.

There were many studies that had investigated the academic performance of students especially at the tertiary level around the world. Those studies were made for many specific reasons that are useful for the educational institution itself.

In [16], there was a total of 117 respondents from Masters in Computer Application program consisting of 63 male students and 54 female students. The data taken from each respondent was their actual university examination result, and the number of subjects taken in the first and second semester purposing on identifying the factors affecting the students academically and formulating a new teaching pedagogy by using a k-means clustering method.

The work presented in [17] shows clearer objectives which is to predict the performance of students at the end of their 4-year studies with a reasonable accuracy by using only their High School Certificate marks and identifying the courses in the first and second years that affects the students' performance at the end of their studies. The students were classified into five classes of graduation marks which are A $(90 \%-$ $100 \%)$, B (80\%-89\%), C (70\%-79\%), D (60\%$69 \%)$, and $\mathrm{E}(50-59 \%)$. As a result, the instructors of certain courses with potential dropout students should implement some policy and give more academic assistance for those who need it.

In contrasts with [18], the data that is related to academic performance was collected such as gender, entry qualification, CGPA, academic programme and cohort. Respondents were classified based on their entry qualification of STPM (19.6\%), Matric (67.3), Diploma (12.6) and STAM (0.5\%). It is necessary to investigate their academic performance based on their entry qualification to review the intake policies. The finding of the study shows that STPM's students perform better than students from matriculation, diploma or STAM.

There were almost no studies involving the SSA model in predicting student academic performance 
and that makes it a solid reason to conduct this study. Although the SSA is widely used in many fields, it is necessary to give a review on applying SSA in the educational field.

\section{Methodology}

\subsection{Data collection}

The data set used in this study were the GPA results for UPSI undergraduate students from the Faculty of Science \& Mathematics consist of six undergraduate programs which are Bachelor of Education in Physics, Biology, Chemistry, Science, Mathematics and Bachelor of Science (Mathematics) with Education. The data were collected by using questionnaires that were distributed to them via online platforms. Total respondent of 228 students were varied from 3rd Semester to 7th Semester whereby, $82.5 \%$ respondents was female and $17.5 \%$ was male. Overall, there were $72.5 \%$ of respondents are from Department of Mathematics, $12.2 \%$ from Department of Chemistry, while $5.7 \%, 6.6 \%$ and $3.1 \%$ of respondents were from Department of Biology, Department of Science and Department of Physics, respectively.

The original data of GPA students' performance are shown in Figure 1. The increase of daily COVID-19 cases forced students to undergo academic activities through their home using online platforms. The blue line indicates the GPA of the semester that student undergoing offline lectures which is face-to-face while the orange line indicates the GPA obtained by students for the next semester which they undergo an online learning. As observed, the lines showing that the GPA obtained by students are mostly increasing from the first academic session to the second academic session. Although some students are dropping, this data can be useful for lecturers to identify and finding ways to assist them in academic. By this data, we can see that the increasing of numbers of students that obtained 4.00 pointer regarding online learning.

The SSA is a model-free approach that can be applied to all types of data, regardless of Gaussian or nonGaussian, linear or nonlinear, and stationary or nonstationary [19]. The student GPA can be decomposed into several additive components via SSA, which could be defined in the forms of trend, seasonal, and noise components [20]. The possible application areas of SSA are diverse [21-23]. The SSA is composed of two complementary stages, known as the stages of decomposition and reconstruction [24]. In this research, the prediction was conducted by using a free software which is $\mathrm{R}$ software, version 4.0.3, under the RSSA package.

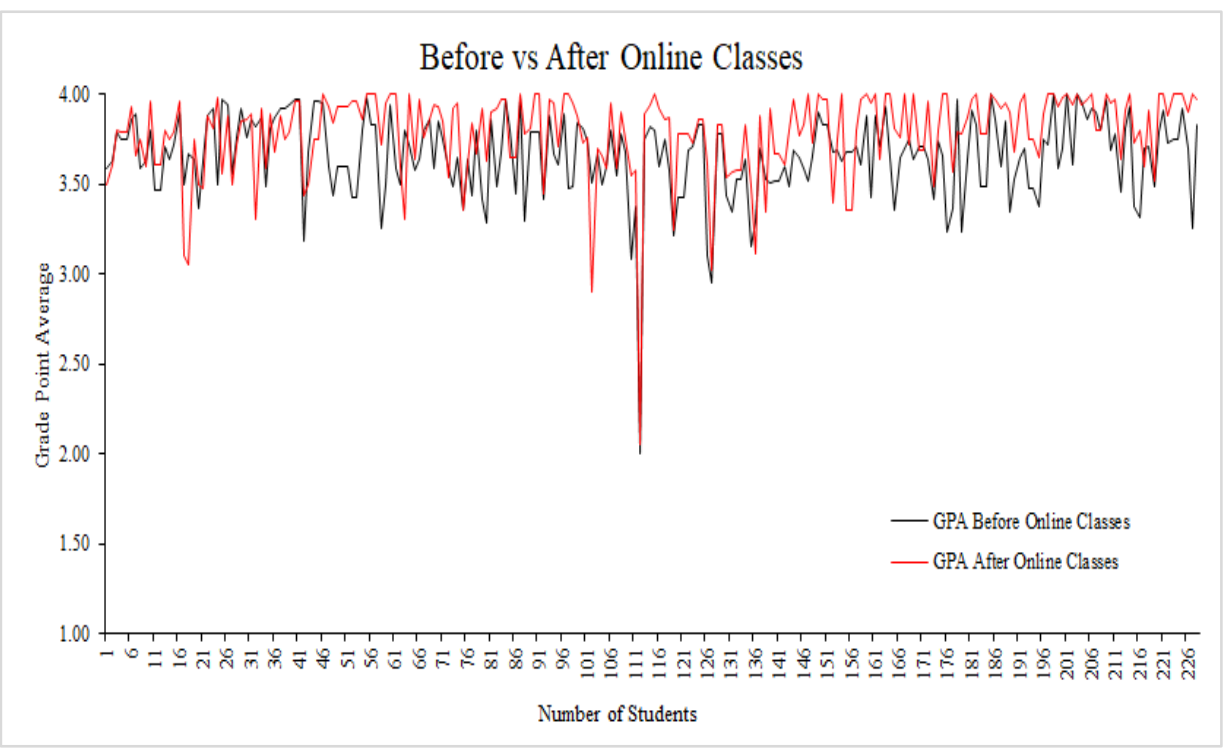

Figure 1 GPA plot before and after online classes for of UPSIs' students 
3.2SSA: decomposition stage

The decomposition stage deviates to two: embedding and SVD. In this stage, the series are decomposed for the attaining of eigen time series data.

Step I: Embedding.

For basic SSA algorithm, the initial step is embedding, which refers to constructing a onedimensional series i.e., univariate vector, $\mathbb{Y}_{T}=$ $\left\{y_{1}, y_{2}, \ldots, y_{T}\right\}$ to a multidimensional series contained in a matrix, $\mathbf{X}=\left(X_{1}, \ldots, X_{K}\right)$, known as the trajectory matrix (see Equation (1)). The rows and columns of $\mathbf{X}$ represent the subseries of the initial onedimensional time series data. The dimension of the trajectory matrix is known as window length, $L$, which ranges at $2 \leq L \leq T / 2$. Columns $X_{1}, \ldots, X_{K}$ of the trajectory matrix, $\mathbf{X}$, are called lagged vectors, $K=T-L+1$.

$\mathbf{X}=\left(X_{1}, \ldots, X_{K}\right)=\left(\begin{array}{ccccc}y_{1} & y_{2} & y_{3} & \cdots & y_{K} \\ y_{2} & y_{3} & y_{4} & \cdots & y_{K+1} \\ y_{3} & y_{4} & y_{5} & \cdots & y_{K+2} \\ \vdots & \vdots & \vdots & \ddots & \vdots \\ y_{L} & y_{L+1} & y_{L+2} & \cdots & y_{T}\end{array}\right)$

Step II: Singular value decomposition (SVD). Secondly, for Step I, the trajectory matrix in is decomposed to obtain the eigen time series formed on their singular values using SVD. The following represents the SVD of the trajectory matrix, $\mathbf{X}$ :

$\mathbf{X}=U^{T} \Sigma V$

where $U=\left(u_{1}, \ldots, u_{L}\right)$ is $L \times L$ orthogonal matrix, $V=\left(v_{1}, \ldots, v_{k}\right)$ is $K \times K$ orthogonal matrix, and $\Sigma$ is $L \times K$ diagonal matrix with non-negative real diagonal entries $\Sigma_{i i}=\sigma_{i}$ for $i=1, \ldots, L$. Vectors $u_{i}$ and $v_{i}$ are left and right singular vectors, respectively, whereas $\sigma_{i}$ refers to singular values. Let $\mathbf{S}=\mathbf{X X}^{\mathbf{T}}$ where singular values are descendingly arranged which is $\left(\sigma_{1} \geq \sigma_{2} \geq \cdots \geq \sigma_{L}\right)$. Let $d=$ $\max \left\{i\right.$, such that $\left.\sigma_{i}>0\right\} . \quad V_{i}=X^{T} U_{i} / \sqrt{\sigma_{i}}(i=$ $1, \ldots, d)$, and the SVD of trajectory matrix, $\mathbf{X}$, is expressed as follows:

$\mathbf{X}=\mathbf{X}_{\mathbf{1}}+\cdots+\mathbf{X}_{\mathbf{d}}$

where $\mathbf{X}_{\boldsymbol{i}}=\sigma_{i} \mu_{i} v_{i}^{T}$. Matrices of $\mathbf{X}_{i}$ are termed as 'elementary matrices', if $\mathbf{X}_{\mathbf{i}}$ has the first rank. Meanwhile, collection $\left(\sigma_{i}, u_{i}, v_{i}\right)$ is the $i$ th eigentriple of SVD.

\subsection{SSA: reconstruction stage}

Grouping and diagonal averaging are the two steps in the reconstruction phase. Here, the original series are reconstructed for future study, including forecasting.

\section{Step I: Grouping.}

Here, the trajectory matrix is divided into two - trend and noise component groups. The set of indices $\{1, \ldots, L\}$ is divided into $m$ disjoint subsets; $I_{1}, \ldots, I_{m}$, based on the division of elementary matrices into groups of $m$. Upon setting $I=\left\{i_{1}, \ldots, i_{p}\right\}$, the resultant matrix, $\mathbf{X}_{I}$, is as follows:

$\mathbf{X}_{I}=\mathbf{X}_{i 1}+\cdots+\mathbf{X}_{i p}$

The retrieved matrices are calculated for $I=I_{1}, \ldots, I_{m}$ and applied in Equation (4). The following presents the expansion:

$$
\mathbf{X}=\mathbf{X}_{I 1}+\cdots+\mathbf{X}_{I m}
$$

where trajectory matrix refers to the total of $m$ resultant matrices. The chosen set of $I=I_{1}, \ldots, I_{m}$ represents eigentriple grouping.

\section{Step II: Diagonal averaging.}

finally, SSA refers to the transformation of each matrix in the grouped decomposition (5) into new series of length, $T$.

Let $\mathbf{Z}$ be $L \times K$ matrix with $z_{i j}, 1 \leq i \leq L$ elements, $1 \leq j \leq K$. Set $\quad L^{*}=\min (L, K), K^{*}=\max (L, K)$, and $N=L+K-1$. Let $z_{i j}^{*}=z_{i j}$ if $L<K$ and $z_{i j}^{*}=z_{j i}$ otherwise. With diagonal averaging, matrix $\mathbf{Z}$ is transferred into $z_{1}, \ldots, z_{T}$ based on the following formula:

$$
z_{k}\left\{\begin{array}{lc}
\frac{1}{k} \sum_{m=1}^{k} z_{m, k-m+1}^{*} & 1 \leq k<L^{*} \\
\frac{1}{L^{*}} \sum_{m=1}^{L^{*}} z_{m, k-m+1}^{*} & L^{*} \leq k \leq K^{*} \\
\frac{1}{T-k+1} \sum_{m=k-k^{*}+1}^{T-K^{*}+1} z_{m, k-m+1}^{*} & K^{*}<k \leq N
\end{array}\right.
$$

Upon employing the diagonal averaging in Equation (6) to the resultant matrix, $\mathbf{X}_{I k}$, reconstructed series of $\widetilde{\mathbb{Y}}_{T}^{(k)}=\left(\tilde{y}_{1}^{(k)}, \ldots, \tilde{y}_{T}^{(k)}\right.$ is produced. The initial series of $\mathbb{Y}_{T}=\left\{y_{1}, y_{2}, \ldots, y_{T}\right\}$ is decomposed into the total of $m$ reconstructed series, $y_{t}=\sum_{k=1}^{m} \tilde{y}_{t}^{(k)}$. The reconstructed series generated by elementary grouping refers to 'elementary reconstructed series.

A key concept when studying SSA is separability that signifies the ways of the varied components of time series may be differentiated to enable future studies. When working with SSA method in numerous study fields, separability becomes a vital mean [25]. The separability impact can result in appropriate decomposition and component extraction. The Wcorrelation technique measures the separability between two distinct components of the reconstructed time series. 
The W-correlation reflects the weighted correlation among components of reconstructed time series that offers highly useful information to both separate and identify groups for the reconstructed components [26]. The elements of the time series terms are indicated by the weights into trajectory matrix. This ranges between 0 and 1 , whereby components that are well separated slant towards 0 , whereas slant towards 1 for otherwise. The $\mathrm{W}$-correlation matrix looks into grouped decomposition among the reconstructed components. The matrix formulation of $\mathrm{W}$-correlation is constructed as:

$\rho_{12}^{w}=\frac{\left\langle X^{(1)}, X^{(2)}\right\rangle w}{\left\|X^{(1)}\right\| w\left\|X^{(2)}\right\| w}$

were,

$\left\|X^{(i)}\right\| w=\sqrt{\left\langle X^{(i)}, X^{(i)}\right\rangle w}, i=1,2,\left\langle X^{(1)}, X^{(2)}\right\rangle w=$ $\sum_{i=0}^{N-1} w_{i} x_{i}^{(1)} w_{i}^{(2)}$,

and weights $w_{i}$ are defined below:

Let $L^{*}=\min (L, K)$ and $K^{*}=\max (L, K)$. As a result,

$w_{i}= \begin{cases}i+1 & \text { for } 0 \leq i \leq L^{*}-1, \\ L^{*} & \text { for } L^{*} \leq i \leq K^{*}, \\ T-i & \text { for } K^{*} \leq i \leq T-1 .\end{cases}$

The graphic illustration of W-correlation is composed of white-black scale, whereby white represents correlation that is small, whereas black denotes correlation between the series components near to value 1

\subsection{SSA: forecasting stage}

\section{Recurrent Forecasting Algorithm}

To perform SSA forecasting, the time series should satisfy the linear recurrent formula (LRF). Time series $Y_{T}=\left(y_{1}, \ldots, y_{T}\right)$ satisfy the LRF of order $\mathrm{d}$ if:

$y_{t}=a_{1} y_{t-1}+a_{2} y_{t-2}+\cdots+a_{d} y_{t-d}$,

$t=d+1, \ldots, T$

In this study, RF-SSA was used for forecasting purpose because it is a popular approach to predict data $[27,28]$. The algorithms described below are detailed in [29]. Let us assume that $U_{j}^{\nabla}$ is the vector of the initial $L-1$ components of eigenvector $U_{j}$, while $\pi_{j}$ represents the final component of $U_{j}(j=$ $1, \ldots, r)$. Denoting $v^{2}=\sum_{j-1}^{r} \pi_{j}^{2}$, coefficient vector $\Re$ is defined as follows:

$\mathfrak{N}=\frac{1}{1-v^{2}} \sum_{j=1}^{r} \pi_{j} U_{j}^{\nabla}$

Upon considering the prior notation, the forecast of $\operatorname{RF-SSA}\left(\hat{y}_{T+1}, \ldots, \hat{y}_{T+M}\right)$ can be attained by $\hat{y}_{i}=\left\{\begin{array}{c}\tilde{y}_{i}, \quad i=1, \ldots, T \\ \mathfrak{N}^{T} Z_{i}, \quad i=T+1, \ldots, T+M\end{array}\right.$

where, $\quad Z_{i}=\left[\hat{y}_{i-L+1}, \ldots, \hat{y}_{i-1}\right]^{T}$ and $\tilde{y}_{1}, \ldots, \tilde{y}_{T}$, represent the values of reconstructed time series that could be retrieved from the previously presented $4^{\text {th }}$ step.

\section{Vector Forecasting Algorithm}

For VF-SSA, the matrix below is considered:

$\Pi=\mathbf{V}^{\nabla}\left(\mathbf{V}^{\nabla}\right)^{T}+\left(1-v^{2}\right) A A^{T}$

where $\mathbf{V}^{\nabla}=\left[U_{1}^{\nabla}, \ldots, U_{r}^{\nabla}\right]$. After that, consider the

linear operator:

$\theta^{(v)}: \mathfrak{Q}_{r} \mapsto \mathbf{R}^{L}$,

Where,

$\theta^{(v)} U=\left(\begin{array}{c}\Pi U^{\nabla} \\ A^{T} U^{\nabla}\end{array}\right)$

Then, the forecast of VF-SSA can be attained by

$Z_{i}=\left\{\begin{array}{cc}\overline{X_{i}} & \text { for } i=1, \ldots, K \\ \theta^{(v)} Z_{i-1} & \text { for } i=K+1, \ldots, K+h+L-1\end{array}\right.$

The detail description for VF-SSA can be found in [30].

\section{Results}

\subsection{Decomposition and reconstruction}

Figure 2 showed the 11 eigenvectors plot obtained from decomposition stage. These plots were then were grouped following its characteristics either noise, trend, or seasonal components. The component of trend was identified from eigenvector plot, in which seasonal and trend components have sine waves indicated by the slow cycles found in the graph (high frequency). Meanwhile, the component of noise was represented by the saw-tooth found in the graph (low frequency). The leading eigenvector has nearly continual coordinates, thus corresponding to a pure smoothing by Bartlett filter [31, 32].

Once the components have been identified, the next process was to reconstruct the eigentriples. The reconstruction result by each of the 11 eigentriples presented in Figure 3. The two figures verified the compatibility of the first and second eigentriple with the trend, whereas the remaining eigentriples had the noise component, thus irrelevant to trend.

Figure 4 demonstrates the components of the reconstructed time series plot from the trend extricated via RF-SSA for the students' GPA. The reconstructed series is the new dataset derived from the original data, which is clear from noise. It is a crucial aspect in SSA to ensure that the forecasting results are precise and accurate [33]. The trend 
component in the time series data was used to observe the occurrence of the trend and pattern, as it was randomly-tabulated as per daily cases.

In Figures 4(a), the trend was precisely generated by a leading eigentriple, which coincided with the initial reconstructed component exhibited in Figure 3. In Figure $4(b)$ the illustration of the trend was precisely generated by both leading eigentriples, which coincided with the first and second reconstructed components shown in Figure 7. The dashed and straight lines on the plot denote the reconstructed series according to the trend component which was extricated from SSA and the GPA student's original time series data, respectively. The plot of reconstructed time series components, produced by both leading eigentriple, abides by the original GPA data although noise component was omitted for $\mathrm{L}=11$ for GPA data at UPSI.

For proper identification of seasonal series components, the graph of eigenvalues and scatterplots of eigenvectors were applied. In order to determine the seasonal series components using eigenvalues plot, several steps were produced by approximately equal eigenvalues. Figure 5 portrays the plot of the logarithms of the 11 singular values for the GPA student's data in UPSI. It clearly showed that no step produced by approximately equal eigenvalues that corresponded to a sine wave. The scatterplot of eigenvectors displays the regular polygons yielded by a pair of eigenvectors to demonstrate that the series components have produced seasonality components. Based on Figure 6 , no pair of eigenvectors produced regular polygons. This confirmed that the GPA results data in UPSI were not influenced by the seasonality since both figures did not have sine wave.

The graphs in Figure 7 illustrate the heat-plot based on W-correlations using the SSA approach. For the reconstructed components, the heat-plot of Wcorrelation based on white-black scale ranges between 0 and 1 [34]. Huge correlation values among the reconstructed components exhibited the possibility of the components to form a group while corresponding to the same component. Importantly, in the extraction of trend, the correlations of trend and noise should be near to zero. At this point, the correlation with $\mathrm{L}=11$ is small, 0.008 which is close to 0 . As illustrated in Figure 7, every square shade stands for the strength W-correlation signifies two components. Subsequently, this denotes that the components of trends are still, to some extent, mixed with the noise and seasonal components in SSA and it was rectified by the window length, $\mathrm{L}=11$, which is evidently demonstrated in Figure 7 for better separability.

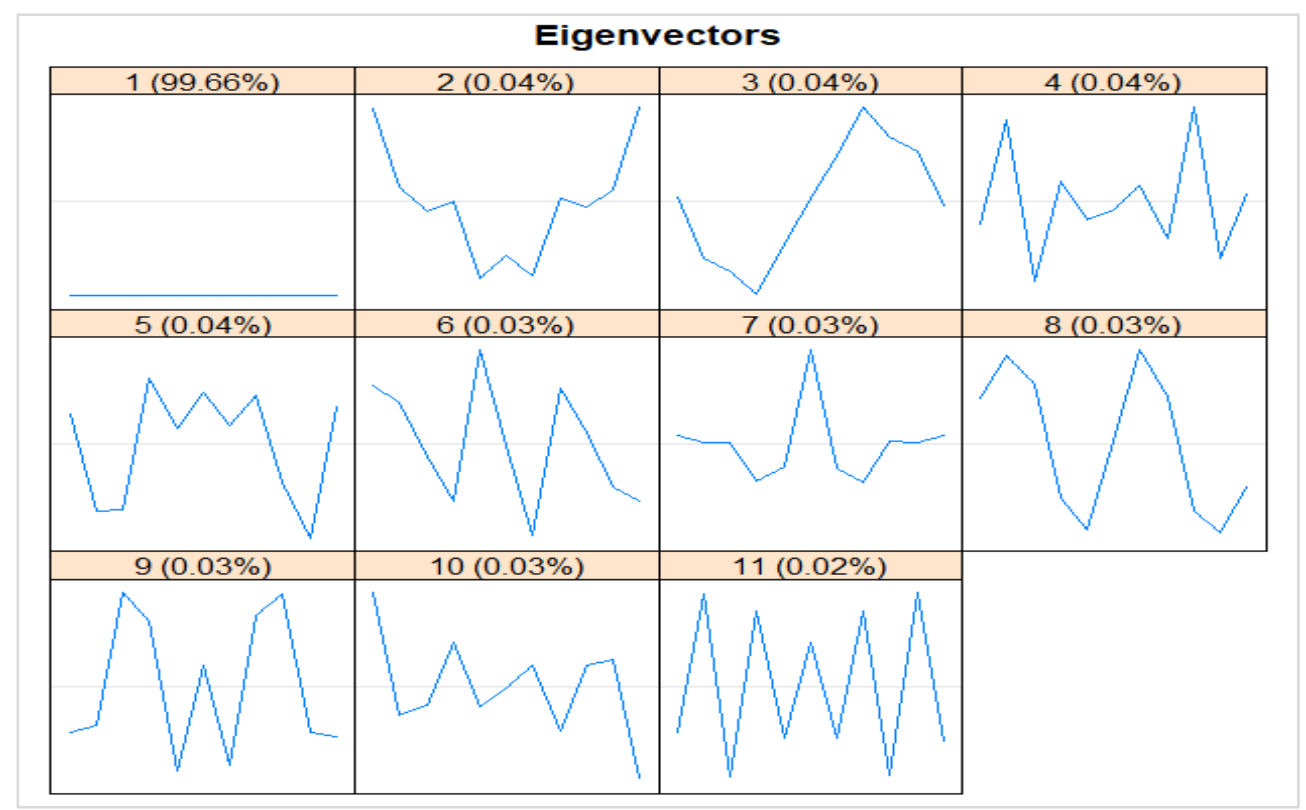

Figure 2 Eigenvectors plot using singular spectrum analysis 
Muhammad Fakhrullah Mohd Fuad et al.

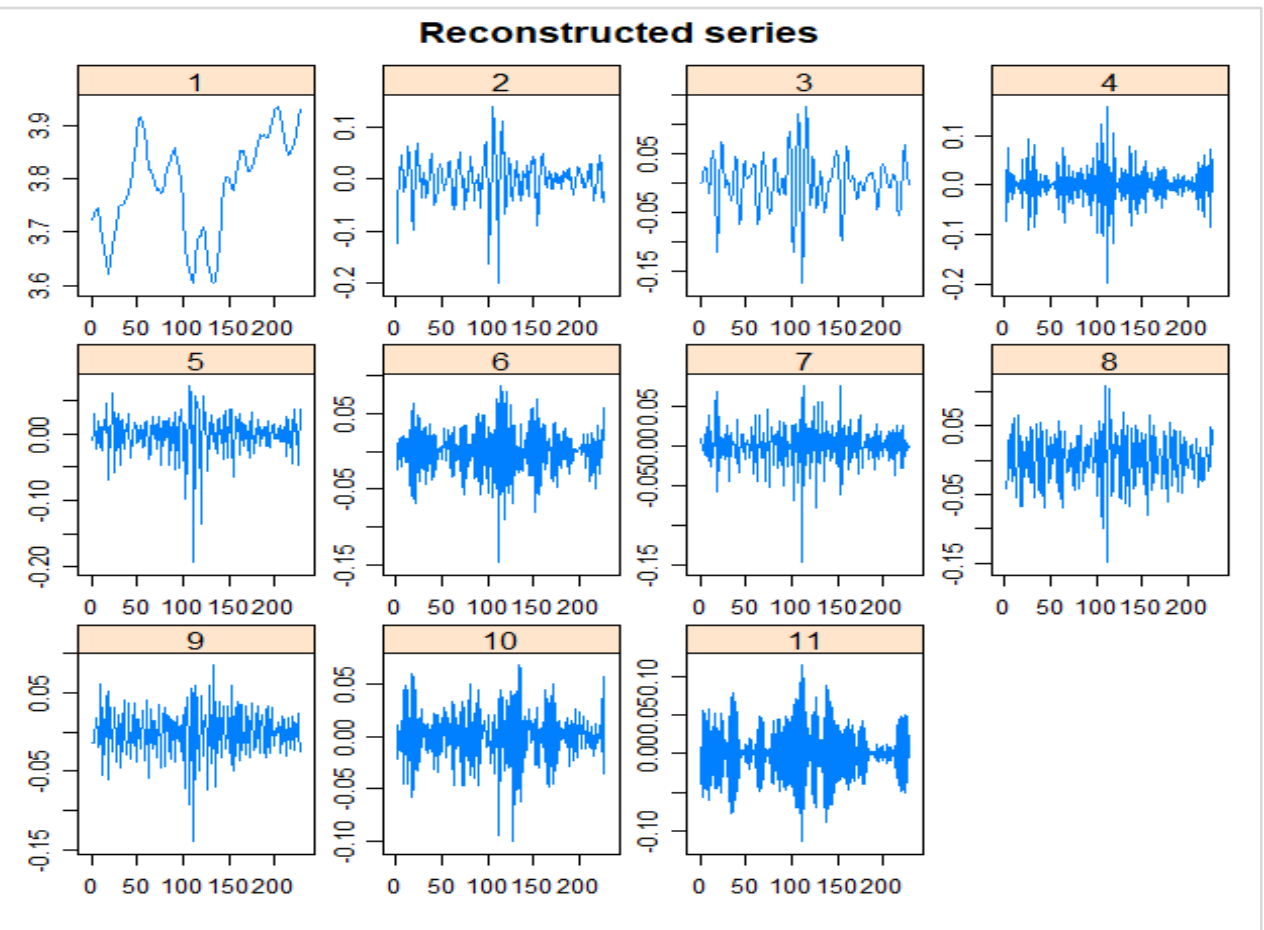

Figure 3 First stage: elementary reconstructed series $(\mathrm{L}=11)$.

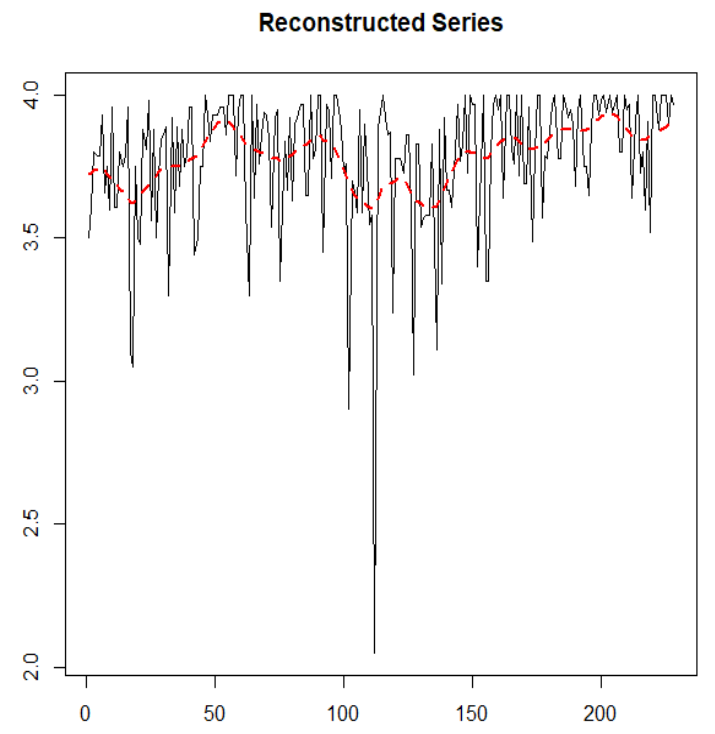

(a)

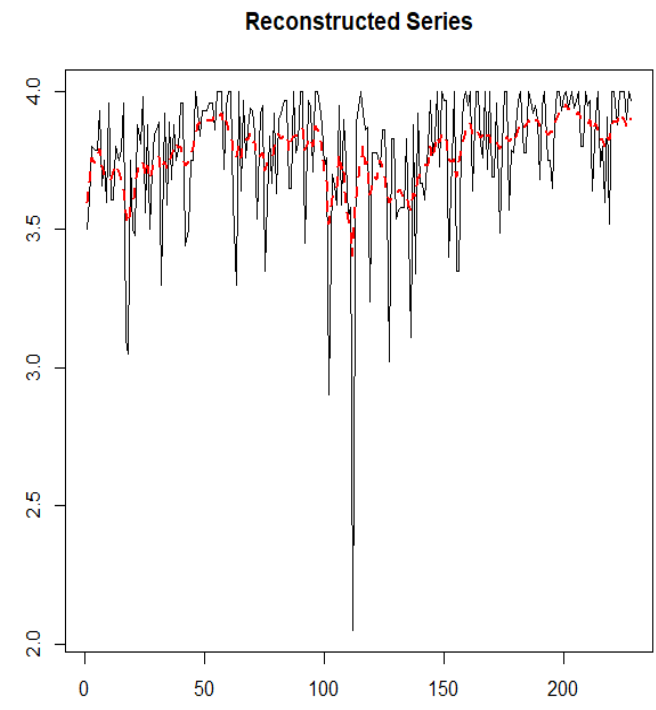

(b)

Figure 4 GPA student's performances of reconstructed components from extracted trends using SSA at L=11. 
International Journal of Advanced Technology and Engineering Exploration, Vol 8(74)

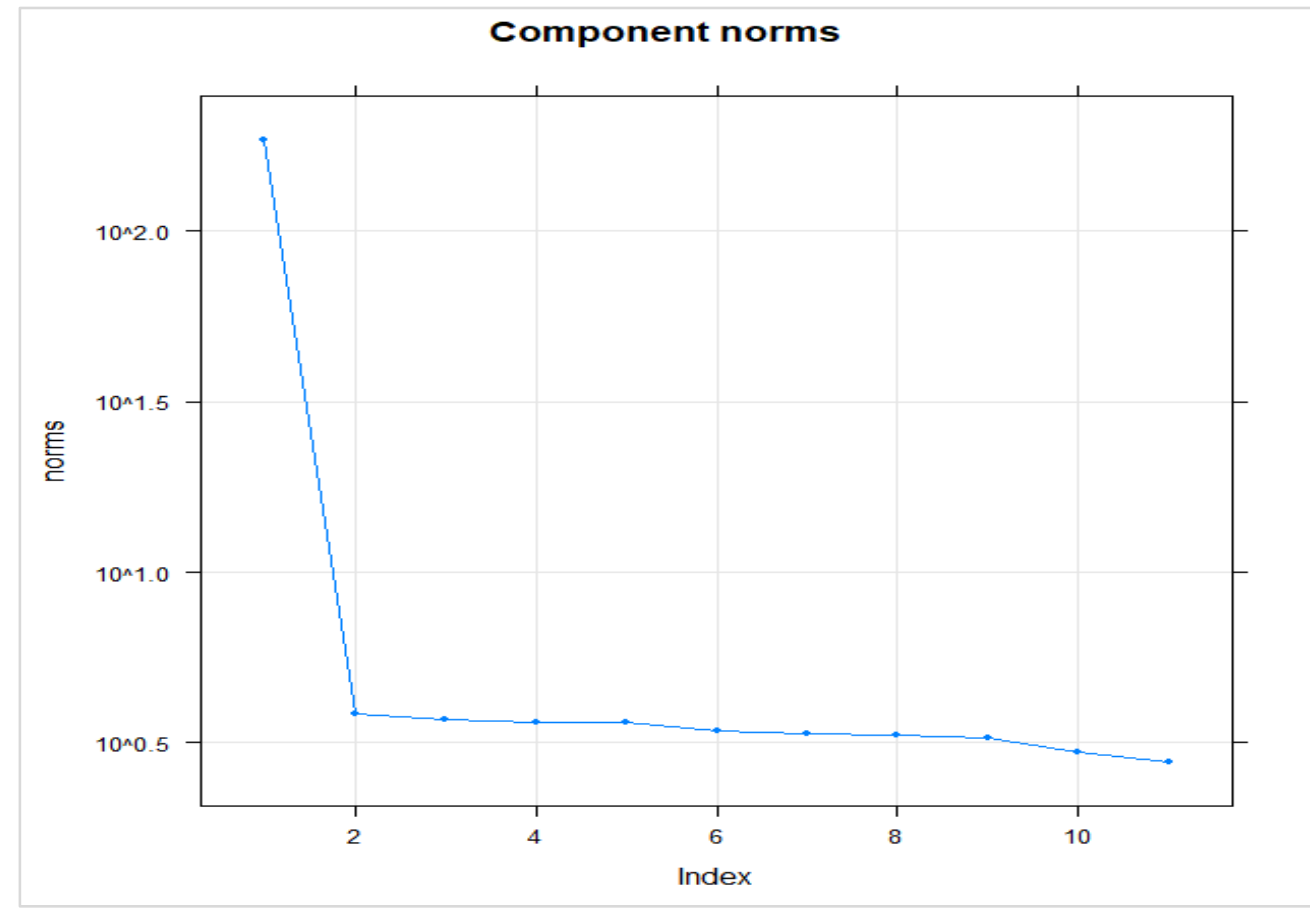

Figure 5 Logarithms of 11 eigenvalues

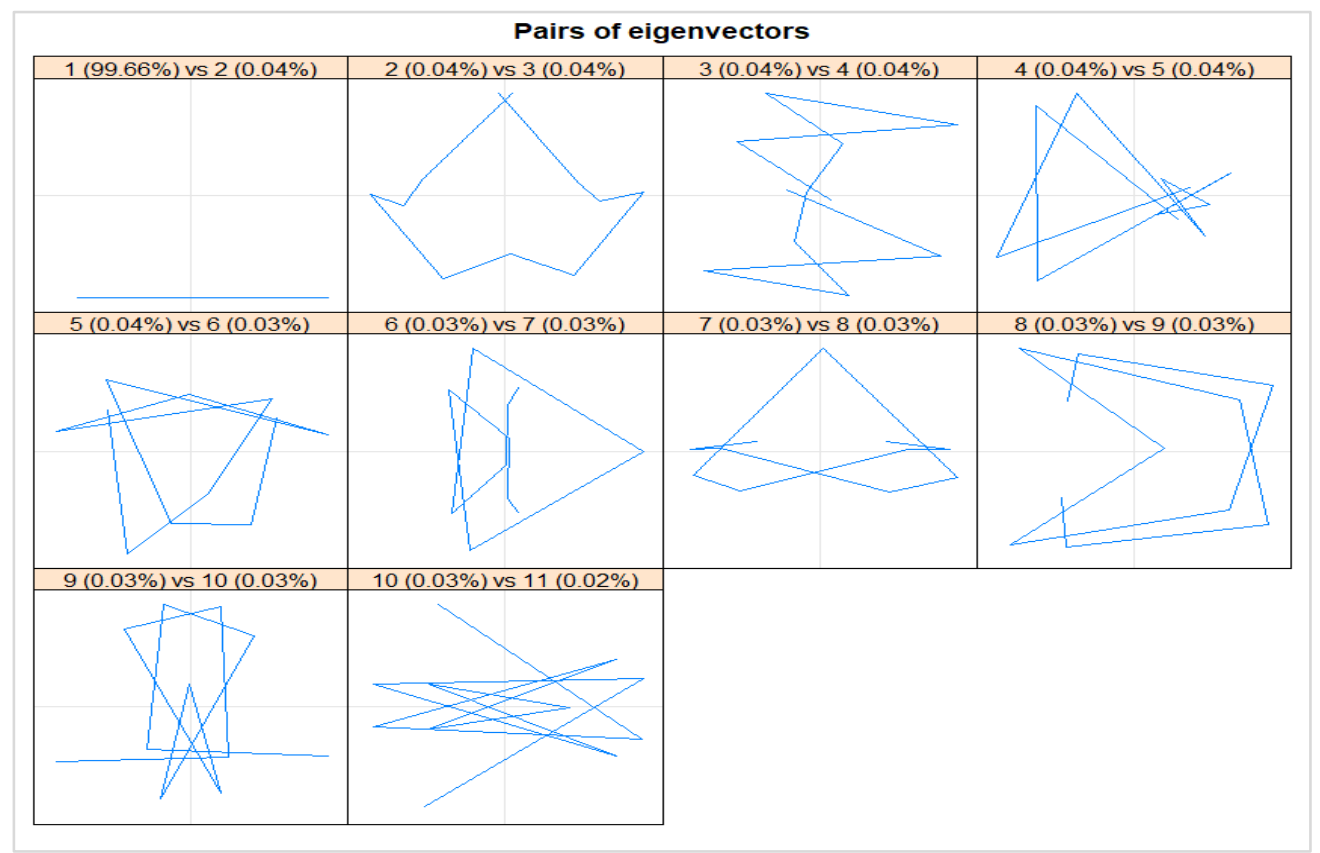

Figure 6 Plots of eigenvectors (EV) pairs for GPA student's data at UPSI 


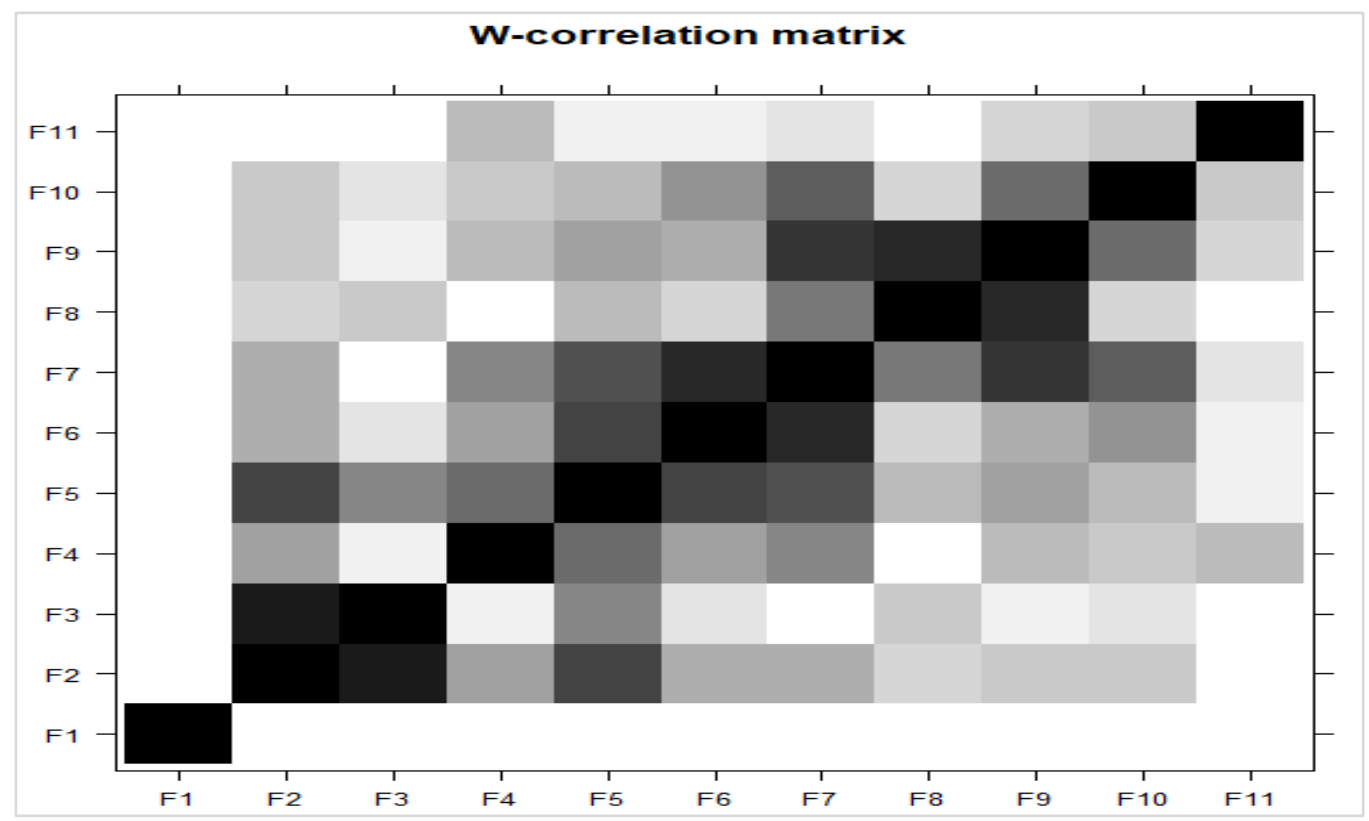

Figure $7 \mathrm{~W}$-correlation plot using SSA with varied windows length, $\mathrm{L}=11$

\subsection{Experiments using RF-SSA and VF-SSA models}

As mentioned in the previous section, the GPA data was first decomposed and reconstructed using SSA model. The next step in this study is to predict the future cases of GPA data in UPSI. In this stage, an SSA forecasting algorithm known as RF-SSA and VF-SSA were applied accordingly. Table 1 presents the performances of SSA forecasting models using two different approaches.

The experimental results clearly showed RF-SSA model performed better than VF-SSA model with the lowest RMSE and the highest $r$ values. Other than that, the results also indicate RF-SSA model tend to slightly under-forecast the student's academic performance with an average absolute error of 0.14 units. This can be compared to VF-SSA model where the model has over-forecast by 0.23 with an average absolute error of 0.22 units. Upon details analysis, it is revealed that VF-SSA, unable to follows the variation in student's academic performance as compared to RF-SSA model. Furthermore, VF-SSA keep increasing its prediction values gradually, proving that the VF forecasting model is more suited to be used with data that has increasing and decreasing trend rather than data that has fluctuations.

Table 1 RF-SSA and VF-SSA prediction performance

\begin{tabular}{lccccc}
\hline SSA Forecasting Models & RMSE & MAE & $\boldsymbol{r}$ & MFE & Decision \\
\hline Vector & 0.30 & 0.22 & 0.21 & -0.23 & $\begin{array}{l}\text { MFE }<0, \text { model tends } \\
\text { to over-forecast }\end{array}$ \\
\hline Recurrent & $\mathbf{0 . 1 9 *}$ & $\mathbf{0 . 1 4 *}$ & $\mathbf{0 . 5 1 *}$ & $\mathbf{0 . 0 1 *}$ & $\begin{array}{l}\text { MFE }>0, \text { model tends } \\
\text { to under-forecast }\end{array}$ \\
\hline
\end{tabular}

*denotes for the lower values

\section{Discussion}

\subsection{Decomposition and reconstruction}

Despite encouraging statistics and findings, it is undeniable that RF-SSA is unable to fully cater the variation in academic students' performance. This can be further proved by analysing Figure 8 . The above figure shows that RF-SSA is unable to predict sudden drop or extreme value which existed in the dataset. This may be due to the fact that the dataset is lacking with extreme data points, making the model unable to catch the underlying pattern. This finding is in agreement with previous researchers stating that RF-SSA model requires large dataset that has clear representation of all possible situations. The study deems that VF-SSA model is unsuitable for catching and predicting data full with variations. The study also found that the size of datasets plays an important role in providing good results for RF-SSA model and 
the model is unable to cater an extreme value in the dataset.

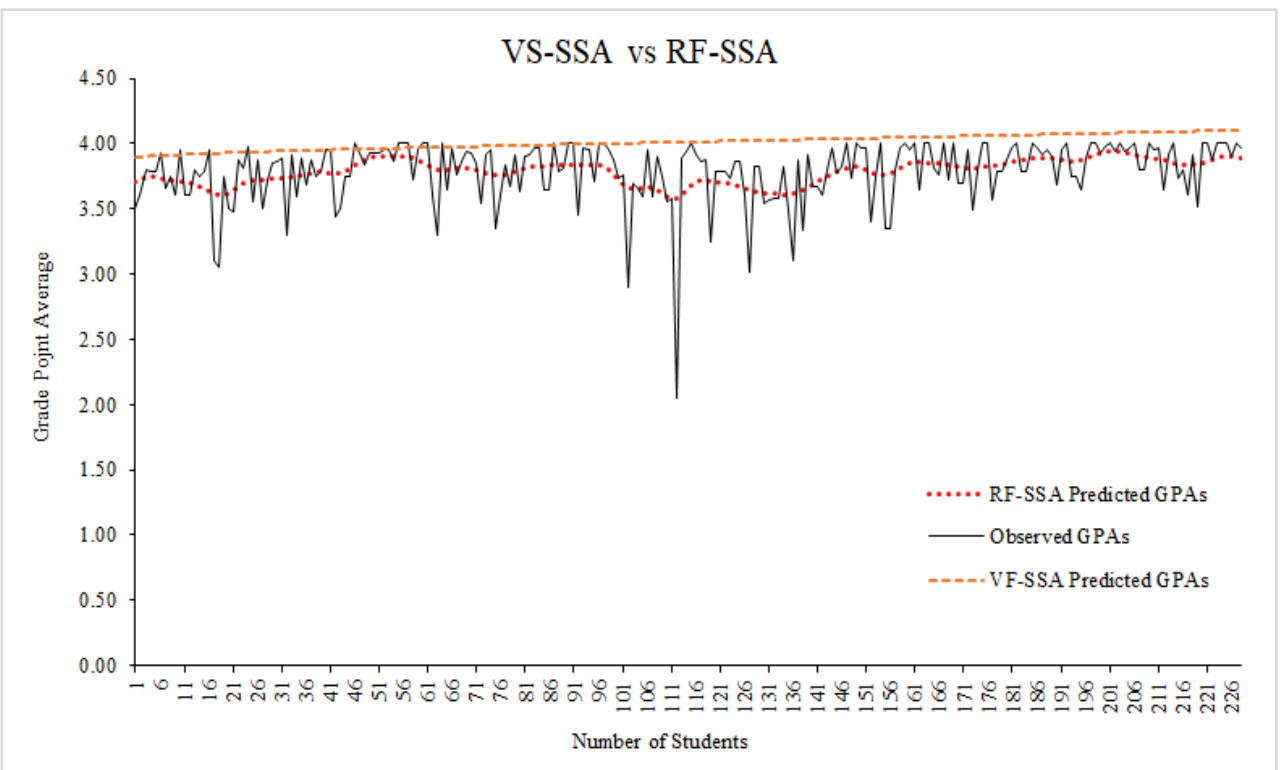

Figure 8 Predicted VF-SSA versus RF-SSA for GPA results for UPSI

\subsection{Limitation of forecasting SSA model}

Some limitations of this study, which should be emphasized when using the forecasting SSA model in assessing the student's academic performance, are as follows:

1. The forecasting SSA models work best when the data exhibit a stable or consistent pattern over time with a minimum amount of outlier. This can help to obtain accurate results for future predictive cases.

2. The forecasting SSA models are mainly used to project future values using historical time series data for short-term forecast.

3. Different observed behaviour of a dataset might influence the selection of window length.

\section{Conclusion and future work}

Overall, the findings in this study brings insight and guideline for lecturers and universities in finding better ways in improving online learning and teaching, preparing suitable online assessment platform, the effective deliver method and etc. It is suggested for future researchers to enhance the RFSSA model so that the model is capable of overcoming its weakness in the current study.

It is also encouraged for future researchers to combine these two models with other prediction models to form a hybrid prediction model such as
Support Vector Machine, Random Forest Classifier, Linear Model, K-Means, and etc. By exploring various type of prediction models, researchers will be able to identify the accuracy, superiority and the weakness of each of these models and can determine which model is best to be used as a guide in predicting students' academic performance from various angles and make a proper conclusion from it.

\section{Acknowledgment}

This research has been carried out under Fundamental Research Grants Scheme (FRGS/1/2019/STG06/UPSI/02/4) provided by Ministry of Education of Malaysia.

\section{Conflicts of interest}

The authors have no conflicts of interest to declare.

\section{References}

[1] https://www.who.int/emergencies/diseases/novelcoronavirus-2019/advice-for-public. Accessed 9 November 2020.

[2] https://www.who.int/emergencies/ diseases/novelcoronavirus-2019. Accessed 9 November 2020.

[3] https://www.malaymail.com/news/malaysia/2020/05/2 7/higher-education-ministry-all-university-lectures-tobe-online-only-until-e/1869975. Accessed 9 November 2020.

[4] http://www.ukm.my/wadahict/pembelajaran-atastalian-epsa-coursera-edxfuturelearn/. Accessed 9 November 2020. 
[5] Bao W. COVID-19 and online teaching in higher education: a case study of Peking University. Human Behavior and Emerging Technologies. 2020; 2(2):1135.

[6] Iyer P, Aziz K, Ojcius DM. Impact of COVID-19 on dental education in the United States. Journal of Dental Education. 2020; 84(6):718-22.

[7] Alvin MD, George E, Deng F, Warhadpande S, Lee SI. The impact of COVID-19 on radiology trainees. 2020; 296(2):246-8.

[8] Mian A, Khan S. Medical education during pandemics: a UK perspective. BMC Medicine. 2020; 18:1-2.

[9] Rose S. Medical student education in the time of COVID-19. JAMA. 2020; 323(21):2131-2.

[10] Kanneganti A, Sia CH, Ashokka B, Ooi SB. Continuing medical education during a pandemic: an academic institution's experience. Postgraduate Medical Journal. 2020; 96(1137):384-6.

[11] Sandhu P, De Wolf M. The impact of COVID-19 on the undergraduate medical curriculum. Medical Education Online. 2020; 25(1):1-2.

[12] https://seleritysas.com/blog/2019/ 12/12/types-ofpredictive-analytics-models-and- how-they work/\#: :text=There $\% 20$ are $\% 20$ several $\% 20$ types $\% 20$ of,algorithms\%20and\%20neural\%20network\%20algor ithms. Accessed 9 November 2020.

[13] Burman I, Som S. Predicting students academic performance using support vector machine. In amity international conference on artificial intelligence 2019 (pp. 756-9). IEEE.

[14] Lau ET, Sun L, Yang Q. Modelling, prediction and classification of student academic performance using artificial neural networks. SN Applied Sciences. 2019.

[15] Hamoud A, Hashim AS, Awadh WA. Predicting student performance in higher education institutions using decision tree analysis. International Journal of Interactive Multimedia and Artificial Intelligence. 2018; 5(2):26-31.

[16] Aggarwal D, Sharma D. Application of clustering for student result analysis. International Journal of Recent Technology and Engineering. 2019; 7(6C):50-3.

[17] Asif R, Merceron A, Pathan MK. Predicting student academic performance at degree level: a case study. International Journal of Intelligent Systems and Applications. 2014:49-61.

[18] Zakaria R, Satari SZ, Damahuri NA, Khairuddin R. Descriptive analysis of students' CGPA: a case study of Universiti Malaysia Pahang. In IOP conference series: materials science and engineering 2019 (pp. 19). IOP Publishing.

[19] Shaharudin SM, Ahmad N, Yusof F. Effect of window length with singular spectrum analysis in extracting the trend signal on rainfall data. In AIP conference proceedings 2015 (pp. 321-6). American Institute of Physics.

[20] Shaharudin SM, Ahmad N, Zainuddin NH. Modified singular spectrum analysis in identifying rainfall trend over peninsular Malaysia. Indonesian Journal of
Electrical Engineering and Computer Science. 2019; 15(1):283-93.

[21] Shaharudin SM, Ahmad N, Mohamed NS, Aziz N. Performance analysis and validation of modified singular spectrum analysis based on simulation torrential rainfall data. International Journal on Advanced Science, Engineering and Information Technology. 2020; 10(4): 1450-6.

[22] Vile JL, Gillard JW, Harper PR, Knight VA. Predicting ambulance demand using singular spectrum analysis. Journal of the Operational Research Society. 2012; 63(11):1556-65.

[23] Alexandrov T, Golyandina N, Spirov A. Singular spectrum analysis of gene expression profiles of early drosophila embryo: exponential-in-distance patterns. Research Letters in Signal Processing. 2008.

[24] De Carvalho M, Rua A. Real-time nowcasting the US output gap: singular spectrum analysis at work. International Journal of Forecasting. 2017; 33(1):18598.

[25] Golyandina N, Shlemov A. Variations of singular spectrum analysis for separability improvement: nonorthogonal decompositions of time series. arXiv preprint arXiv:1308.4022. 2013.

[26] Golyandina N, Korobeynikov A. Basic singular spectrum analysis and forecasting with R. Computational Statistics \& Data Analysis. 2014; 71:934-54.

[27] Danilov DL. Principal components in time series forecast. Journal of Computational and Graphical Statistics. 1997; 6(1):112-21.

[28] Danilov D. The caterpillar method for time series forecasting. Principal Components of Time Series: The Caterpillar Method. 1997:73-104.

[29] Hassani H, Mahmoudvand R. Multivariate singular spectrum analysis: a general view and new vector forecasting approach. International Journal of Energy and Statistics. 2013; 1(01):55-83.

[30] Ghodsi M, Hassani H, Rahmani D, Silva ES. Vector and recurrent singular spectrum analysis: which is better at forecasting? Journal of Applied Statistics. 2018; 45(10):1872-99.

[31] Golyandina N, Nekrutkin V, Zhigljavsky AA. Analysis of time series structure: SSA and related techniques. CRC Press; 2001.

[32] Mahmoudvand R, Alehosseini F, Rodrigues PC. Forecasting mortality rate by singular spectrum analysis. REVSTAT-Statistical Journal. 2015; 13(3):193-206.

[33] Dong Y, Zhang L, Liu Z, Wang J. Integrated forecasting method for wind energy management: a case study in China. Processes. 2020; 8(1):1-26.

[34] Shaharudin SM, Ismail S, Tan ML, Mohamed NS, AininaFilzaSulaiman $\mathrm{N}$. Predictive modelling of covid-19 cases in Malaysia based on recurrent forecasting-singular spectrum analysis approach. International Journal of Advanced Trends in Computer Science and Engineering. 2020; 9(1.4):175-83. 


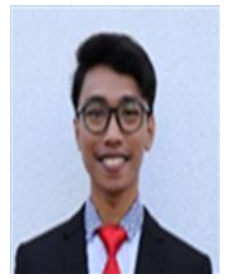

Muhammad Fakhrullah Mohd Fuad is a student from Universiti Pendidkan Sultan Idris (UPSI) majoring in Bachelor of Science (Mathematics) with Education. Since UPSI has been conducting online learning since March 2020, he intends to predict the academic performance of his peers throughout online learning to help lecturers and educators improvised existing online learning and teaching methods. Email: d080939@siswa.upsi.edu.my

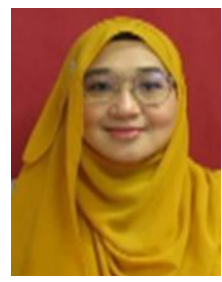

Shazlyn Milleana Shaharudin as born in Johor Bahru, Malaysia, in 1988. She is a senior lecturer at the Department of Mathematics, Faculty of Science and Mathematics, Universiti Pendidikan Sultan Idris (UPSI). She graduated with a bachelor science degree in Industrial Mathematics and Doctor of Philosophy (Mathematics) from Universiti Teknologi Malaysia. During her $\mathrm{PhD}$ journey, she developed an interest in multivariate analysis, specifically in finding patterns which deals with big data. Her research focuses on the area of Dimension Reduction Methods specifically in Climate Informatics which involves analysis on huge climate-related datasets based on techniques in Data Mining. She had published her research in Scopus indexed journal and presented her work in various local and international conferences.

Email: shazlyn@fsmt.upsi.edu.my

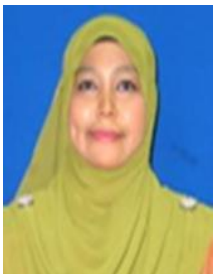

Shuhaida Ismail is a lecturer at the Department of Mathematics and Staticstics, Faculty of Applied Sciences and Technology, Universiti Tun Hussein Onn Malaysia (UTHM). She obtained her first degree in Computer Sciences majoring from UTM. She also obtained a Master degree and $\mathrm{PhD}$ from the same university. Throughout her studies, she developed an interest in Machine Learning research area, specifically in Predictive Modelling, Classification, and Clustering. Her current research areas are in Hydrological Modelling, Big Data Analytics and Deep Learning.

Email: shuhaida@uthm.edu.my

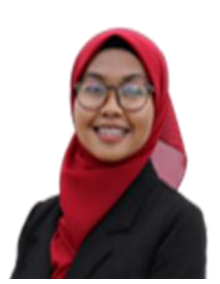

Nor Ain Maisarah Samsudin is a student from Universiti Pendidikan Sultan Idris (UPSI) majoring in Bachelor of Science (Mathematics) with Education. Since UPSI has been conducting online learning since March 2020, she intends to predict the academic performance of her peers throughout online learning to help educators improvised existing online learning and teaching methods.

Email: d080938@siswa.upsi.edu.my

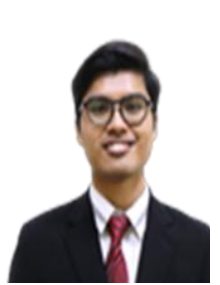

Muhammad Fareezuan Zulfikri is a student from Universiti Pendidikan Sultan Idris (UPSI) majoring in Bachelor of Science (Mathematics) with Education. Since UPSI has been conducting online learning since March 2020, he intends to predicts the academic performance of his peers throughout online learning to help educators improvised existing online learning and teaching methods.

Email: d080937@siswa.upsi.edu.my 